\title{
Féeries
}

Études sur le conte merveilleux, XVII $-\mathrm{XIX}{ }^{\mathrm{e}}$ siècle

\section{Dans quel sens les Mille et une nuits et les féeries classiques sont-elles « comiques»?}

Jean-Paul Sermain

\section{(2) OpenEdition}

1 Journals

Édition électronique

URL : http://journals.openedition.org/feeries/593

DOI : $10.4000 /$ feeries.593

ISSN : 1957-7753

Éditeur

UGA Éditions/Université Grenoble Alpes

Édition imprimée

Date de publication : 1 juin 2008

Pagination : 25-31

ISBN : 978-2-84310-123-6

ISSN : $1766-2842$

\section{Référence électronique}

Jean-Paul Sermain, «Dans quel sens les Mille et une nuits et les féeries classiques sont-elles « comiques »? », Féeries [En ligne], 5 | 2008, mis en ligne le 01 septembre 2009, consulté le 08 septembre 2020. URL : http://journals.openedition.org/feeries/593 ; DOI : https://doi.org/10.4000/ feeries.593

Ce document a été généré automatiquement le 8 septembre 2020

(c) Féeries 


\title{
Dans quel sens les Mille et une nuits et les féeries classiques sont-elles « comiques»?
}

\author{
Jean-Paul Sermain
}

LES AFFINITÉS CONTEMPORAINES et les usages de lecture relèvent plutôt, dans les contes de fées ou les récits merveilleux comme ceux des Mille et Une Nuits, leur appel à l'imagination, à la sensibilité, à la fantaisie ils nous introduisent dans un monde surréel, ils parlent aux enfants, ils parlent au cœur. Triple effet de leur appropriation romantique, de leur interprétation par la psychanalyse, et de leur intégration à ce qu'on appelle vilainement la «littérature jeunesse». Leur aspect comique n'attire guère l'attention et le relever peut paraitre sinon incongru du moins déplacé. Le responsable de ce numéro Jean Mainil a pourtant intitulé son ouvrage important sur les contes de $\mathrm{M}^{\mathrm{me}} \mathrm{d}^{\prime}$ Aulnoy «le rire des fées ${ }^{1}$ »; et j'ai moi-même abordé deux fois cette question dans un numéro d'Humoresques consacré aux liens du risible et de l'horrible ; et dans un chapitre de mon livre de poétique historique, Le Conte de fées du classicisme aux Lumières ${ }^{2}$. On a tous en mémoire des situations, des épisodes ou des détails drôles qu'une petite fille venue apporter un petit pot de beurre prenne sans hésitation un loup couché à la place de sa grand-mère pour sa grand-mère même, qu'un cocher porte une moustache parce qu'il est issu d'une souris, qu'une reconnaissance familiale, au terme de traverses multiples, se fasse par l'intermédiaire d'une tarte à la crème, que, dans la pauvre demeure d'Aladdin et de sa mère, il suffise de frotter une lampe pour qu'un génie en surgisse, qu'il faille faire appel à un cordonnier pour recoudre le corps de son frère morcelé par des brigands et ainsi l'enterrer ni vu ni connu. On pourrait d'emblée tirer de ces six exemples pris au hasard ${ }^{3}$ une remarque sur une des singularités de cette drôlerie elle vient de l'interférence entre des circonstances très familières, banales, prosaïques (l'apparence d'un cocher venu vous conduire au bal; la grand-mère alitée dans sa chaumière ; l'envie de manger une sucrerie dans un voyage ; une mère qui fait le ménage ; les compétences de celui qui sait coudre les peaux), mais devenues bizarres, ridicules, burlesques (qualifications ici avancées pour rendre compte d'une impression et sur lesquelles il nous faudra revenir), par le caractère 
aberrant, surnaturel, merveilleux de l'univers dans lequel ces événements prennent place les loups parlent aux jeunes filles qui ne s'en étonnent pas et peuvent passer pour des grands-mères qu'ils viennent de dévorer ; une fée vient vous tirer de votre abandon en vous fournissant de quoi aller au bal en métamorphosant ce que la campagne fournit de potiron et de mulots; sept filles d'ogre portant des couronnes en dormant et un petit garçon se sauve en remplaçant ces couronnes par les bonnets de ses frères; des génies ont substitué le jour de la nuit de noces à un promis contrefait le plus beau des garçons dont les étreintes ont été fécondes et l'ont ensuite ramené à l'autre bout de l'Orient, laissant la fille mère chercher le géniteur ; une vieille lampe insignifiante qui, au moment où on la nettoie, fait surgir un génie prêt à vous donner tout ce que vous voulez et à qui vous ne demandez qu'un bon repas; une formule magique permet d'entrer dans la caverne où les voleurs entreposent leurs trésors, mais elle est oubliée par le frère du héros au moment où il veut ressortir les bras chargés d'or, et voilà qui vous condamne à mort et oblige Ali Baba à se débarrasser au mieux du cadavre...

2 Ce qui apparaît est donc que le « comique » des contes merveilleux et féeriques tire ses particularités des propriétés mêmes du genre, c'est-à-dire d'un traitement du merveilleux au sein de l'histoire. Ce sont ces deux éléments qui doivent nous guider dans notre réflexion. Pour caractériser le "conte", il est utile de renoncer à une définition essentialiste, qui nous conduirait à prendre en compte des textes proches de la nouvelle fantastique, horrible, tragique, et de nous en tenir à sa perception au tournant des $\mathrm{XVII}^{\mathrm{e}}$ et $\mathrm{xVIII}{ }^{\mathrm{e}}$ siècles (et la même observation vaut encore au moins jusqu'au milieu du XVIII $)$ et, si l'on peut dire, à sa position au sein des récits fictionnels récit assez court (la grande longueur devenant un écart), dans lequel les personnages et l'intrigue se manifestent essentiellement par des actions, et surtout ouvertement fictif (alors que le roman vise alors à justifier par une impression de vérité sa propre énonciation), ce qui se manifeste souvent par sa prise en charge explicite par un " conteur », par l'introduction du merveilleux et, ce qui nous importe plus ici, parce qu'il est "fait à plaisir», qualification pas toujours présente bien entendu mais implicitement contenu dans le "contrat " du texte, valant pour son auteur et son lecteur et les assez nombreuses figurations que le conte aime à donner de ces deux instances. On pourrait certes dire que toute littérature est «faite à plaisir » puisqu'elle nous éloigne des tâches plus essentielles de la vie ou de la religion; mais l'expression a néanmoins un sens plus étroit, dont le terme « divertissant » employé par Galland pour qualifier les contes des Nuits rend assez bien compte ${ }^{4}$ renoncements aux ambitions civiques, poétiques, historiques de la littérature, recours à une invention " agréable ", capable de "plaire » et de « distraire » (il n'est pas étonnant que le conte fournisse des matériaux au cinéma de masse). Cette restriction ou cette modestie ont pu prendre vers 1690 une valeur "subversive" au regard et du sérieux monarchique et du sérieux littéraire au moins revendiqué. Certes la littérature sérieuse admet un département comique, qui peut accueillir des éléments frivoles ou burlesques, mais dont la visée faire rire des ridicules, des hypocrisies, des vices - lui sert de justification castigat ridendo mores. Si l'on revient à nos six exemples de contes, la portée critique n'est pas absente, liée à des traits particulièrement conventionnels. Le frère d'Ali Baba et sa femme se sont engagés dans la triste aventure de la caverne aux trésors par ruse, esprit de lucre et sottise ils sont punis. Cendrillon subit la persécution de ses demi-sœurs et son succès auprès du Prince prépare leur châtiment. La petite fille aurait dû savoir qu'il est dangereux de parler au loup et les femmes qui l'ont élevée auraient dû l'en avertir autant de coupables. Les amours d'une nuit des deux jeunes gens sont le résultat d'une 
série d'emportements et de manques de réflexion et de sagesse de leurs parents il faudra bien du temps pour remédier à toutes ces erreurs. Aladdin est un vaurien qui ignore tout du prix des choses, mais qui est par-là exempt de la brutalité infanticide du magicien, inquiétant roi des aulnes. Tout ceci nous incite à retrouver dans les contes les traits du comique qui sert à définir un pôle de la poétique des genres, caractérisé par un mode d'écriture et de perception, mais aussi par des types de personnages et de situations, d'intrigues et une fonction propre (corriger en ridiculisant les mœurs). Le partage, conçu d'abord pour le théâtre (faisant de la "comédie » la référence majeure du comique), s'était appliqué aux romans, et, dans ce transfert, s'était particularisé «le roman comique " devrait être écrit comme un seul nom pour désigner un type de romans fortement codifié dans ses contenus et dans sa relation critique et parodique à l'égard de son alter ego, lui dépourvu de nom et qu'on désignera par commodité le roman hérö̈que ${ }^{5}$ le comique y était proche du grotesque, supposait une dégradation des personnages et des univers représentés, et visait la prétention à la noblesse de l'autre roman, que Thomas Pavel a qualifié d'« idéaliste ${ }^{6} »$. On sait que cette division du champ romanesque s'efface vers 1660 , ce qui implique que le sérieux et le pathétique ne se confondent plus avec le noble et l'héroïque et que le registre, le propos, la veine « comique » du roman s'éloignent du « roman comique » et doivent trouver leur voie propre nous y trouvons par exemple Gil Blas, Le Paysan parvenu ou Faublas. L'une des composantes de la liberté qu'on a invoquée pour expliquer l'essor du roman au XvIII siècle, est justement d'avoir rompu avec la division du comique et du tragique, et donc de s'aventurer dans des registres moyens et surtout dans des compositions mixtes Les Illustres françaises, La Vie de Marianne, Jacques le fataliste, Les Liaisons dangereuses non seulement passent des rires aux larmes mais savent faire percevoir un même moment dans les deux perspectives du plaisant et du pathétique (et même du comique et du tragique).

3 Le conte de fées et le récit merveilleux se situent à peu près dans la première période d'exploration de ces nouveaux genres et registres et mélanges, et ils y contribuent. Leur caractère « divertissant » les place dans la zone comique, une fois entendu que celle-ci a perdu ses frontières. Le « comique » peut donc s'y mêler à un sérieux qui n'est plus nécessairement celui de la critique des ridicules et des vices, mais aussi à une culture de l'imagination et à la peinture de relations affectives et sentimentales. Tout conte merveilleux est comique parce qu'il installe un univers improbable où les éléments les plus anodins s'insèrent dans une logique inédite, se combinent différemment tout en gardant les propriétés les plus élémentaires de leur réalité propre la lampe d'Aladdin reste une lampe quelconque, la tarte à la crème est faite selon une recette spéciale qui assure la renommée du pâtissier, il faut bien enterrer les cadavres selon les normes religieuses et sociales en vigueur (et non en morceaux, ballotant dans le cercueil), on ne peut pas aller au bal du prince à pieds nus (il faut carrosse et chaussures). Le conte de fées, le récit merveilleux vous plongent d'emblée dans un monde à part où les loups parlent, où les voyageurs rencontrent des génies, où le noyau d'olive qu'on jette risque de blesser un être invisible et tout puissant ${ }^{7} . .$. Ils nous invitent à suspendre l'esprit de sérieux, d'autant plus que le surnaturel est presque toujours mis au service de la satisfaction des désirs et de la réparation des maux ${ }^{8}$. On pourrait donc considérer que le caractère ouvertement "comique» de certains épisodes ou détails (paroles ou circonstances de l'intrigue) accentue, souligne, marque ce qui appartient au registre ou à la modalité du conte "divertissant». Cette valeur d'intensification interne est d'autant mieux qualifiée qu'elle procède justement de la réunion du merveilleux et du 
prosaïque, du surnaturel et du naturel, qui définit le genre réunion qui produit un choc " comique » et qui assure la perception des propriétés inhérentes à ce mode de récit et au genre. C'est dans des détails concrets que se manifeste la double appartenance des héros à un monde merveilleux et au monde réel la lampe d'Aladdin, le corps à recoudre par un cordonnier, le carrosse issu d'une citrouille, le loup dévoreur mais capable de passer pour une grand-mère, une tarte si exceptionnelle qu'elle réunit les amants séparés par des génies et emportés sur des sortes de tapis volants. On peut considérer à cet égard que la proximité avec des intentions satiriques sert plutôt à soutenir la perception comique qu'elle n'en assure la signification (elle me paraît secondaire).

Ces six exemples peuvent également nous guider pour saisir une seconde caractéristique du "comique " dans les contes de fées et les récits merveilleux les éléments qui servent de support à la disconvenance des logiques ont des propriétés dont la rencontre ne produit pas seulement un effet comique, mais associent à cet effet comique un aspect bizarre, inquiétant, dérangeant, voire fantastique. Le cadavre découpé du frère qu'il faut tenter de rendre convenable à un enterrement, le loup rusé qui dévore une grand-mère puis une petite fille; la famille éclatée, la mère célibataire, le fils retrouvé en pâtissier...; le voyou récompensé par une lampe; la jeune fille martyrisée, reléguée dans les cendres de la cheminée, devant attendre des fées qu'elles transforment la citrouille en carrosse ; le petit poucet substituant les bonnets de ses frères et les couronnes des filles de l'ogre, suscitant ainsi un infanticide collectif. Voilà les supports du comique; le comique consiste à prendre sur ces réalités affreuses un point de vue amusé (on dirait anachroniquement d'humour et parfois d'humour noir), c'est-à-dire qu'il amène le lecteur à mêler le sentiment d'horreur ou de dégoût ou de pitié à un plaisir, à un sourire. Le lien de cette association avec le genre du conte donne à ces moments de trouble comique une portée réflexive plus étendue c'est en quelque sorte le plaisir même du conte qui naît de cette perturbation et de cette autorisation à rire du pire. Sans doute toutes les occurrences d'intensification comique de la modalité divertissante du conte, ne produisent pas un tel effet (beaucoup sont plus anodinement plaisantes), mais elles sont assez remarquables et fortes, et surtout elles s'inscrivent assez dans la logique d'une écriture, pour qu'elles puissent passer pour leur propriété, pour ce qui la définit elles sont conformes à sa logique profonde. Dans nos six exemples, on peut relever les implications du trouble qu'ils font naître. Le lien entre affection et dévoration dans le petit chaperon rouge mais aussi entre sexualité et destruction est au centre de la coïncidence comique du loup et du trompeur (il peut passer pour la grandmère, il peut pénétrer la jeune fille). Le cadavre du frère à coudre fait apparaître les dangers de la situation d'Ali Baba (qui, sous couvert de son humble position, cherche à rivaliser avec le grand banditisme) mais aussi l'ambivalence de sa position morale et sociale il prend la place des voleurs (il les vole), et entend en même temps se soustraire aux conséquences du vol, respecter les interdits et les conventions. Le petit Poucet n'échappe à plusieurs tentatives de meurtre qu'en préparant des assassinats puis il s'enrichit en servant d'intermédiaire adultère. Chez Aladdin le succès procède directement des transgressions. Dans le cas de la tarte, elle assure la permanence de la transmission familiale sur un mode anecdotique et son absurdité répond à l'absurdité des causes qui l'ont interrompue. Ce qui est en jeu dans ces épisodes où la magie et les pouvoirs surnaturels viennent se glisser dans le prosaïque - à un moment clef de l'intrigue et de l'itinéraire des personnages - c'est la révélation d'un désir ou d'une intention fortement transgressive Ali Baba joue avec le cadavre comme avec la loi, le séducteur-loup détruit les jeunes filles qui se laissent séduire, la tarte rétablit ce que la 
folie des hommes a provoqué, le petit poucet détourne le désir de meurtre sur les autres. Le dernier exemple moins inquiétant, celui de la citrouille, est comparable à celui de la lampe d'Aladdin dans les deux cas, les personnages aux marges sociales connaissent une réussite inouie, absolue. La citrouille comme la lampe qui est longtemps passée inaperçue d'Aladdin servent à marquer la distance infinie des deux états, à inscrire dans la réussite présente la trace d'une origine inverse Aladdin on le sait, à cause de cette même lampe que le magicien parvient à récupérer en jouant de son aspect originel, perd tout, revient à la case départ (il risque même la mort). La lampe et la citrouille sont comiques en ce qu'ils sont à la fois l'origine de la réussite et son contraire radicaux, appartiennent aux deux espaces du prosaïque et du merveilleux ce que raconte le conte de fées c'est la substitution de l'un à l'autre, immédiate, sans motif sinon la grâce, le bon plaisir, la force enfantine du souhait les contes sont faits « à plaisir » en ce qu'ils font accéder à la réalité la loi du plaisir, ou du moins celle du désir ; le détail comique signifie cet infini du désir et d'un univers qui se plie à sa loi. L'étrangeté de l'épisode rend l'étrangeté de ce désir, d'autant plus fortement quand il se mêle à des situations de violences, de rapts et de meurtres désir primitif de destruction autant que d'acquisition, de jouissance immédiate, de revanche. Les moments d'intensification comique poussent la logique du conte à l'extrême (le choc des registres), et l'ébranlement du plaisir (rire ou sourire) fait jouer aussi le caractère trouble de la situation (dans le conte) comme celle du lecteur découvrant une logique de toute puissance et de toute satisfaction ou la nécessité de répondre aux menaces par des violences égales à moins de disparaître.

Le comique du conte est à la fois totalement divertissant, il est l'hyperbole du divertissant, mais en même temps il touche au sérieux par ses implications. Notons qu'il ne cherche pas à donner de leçon sur la société comme la comédie ou la satire (même si le conte parfois en emprunte les outils ou les couleurs), mais c'est une expérience de lecture, les affects mêlés qu'elle suscite (de comique et de gêne, de dégoût et de rire), qui sert de support à une réflexion réflexion sur le genre et sur la position du lecteur (dimension pragmatique essentielle du conte de fées), mais aussi réflexion sur soi : c'est en tant que lecteur amateur qu'il accède à une sorte de vérité morale, c'est une vérité de la lecture et de la consommation des contes.

\section{NOTES}

1. Jean Mainil, Madame d'Aulnoy et le rire des fées, Paris, Kimé, 2001.

2. Jean-Paul Sermain, Le Conte de fées du classicisme aux Lumières, Paris, Desjonquères, 2005, chap. VI, Distances critiques, un esprit comique ; « Horrible et risible, les voies de la transgression dans le conte de fées et la littérature des Lumières ", Humoresques 14, 2001, p. 149-164.

3. Les trois premiers sont dus à Perrault (Le Petit Chaperon rouge, Cendrillon, Le Petit Poucet); les trois autres à Galland dans Les Mille et Une Nuits (Histoire de Noureddin Ali, Histoire d'Aladdin et la lampe merveilleuse, Histoire d'Ali Baba et de quarante voleurs exterminés par une esclave). 
4. Dans la dédicace à la marquise d'O, Galland annonce des narrations «agréables et divertissantes "; il reprend l'expression dans l'Avertissement pour affirmer la supériorité des Arabes dans ce genre.

5. Voir Jean Serroy, Roman et réalité. Les histoires comiques au XVII siècle, Paris, Minard, 1981 et René Démoris, Le Roman à la première personne, du classicisme aux Lumières, Genève, Droz, 2002 (1975).

6. Thomas Pavel, La Pensée du roman, Paris, Gallimard, 2003.

7. C'est ce qui arrive au héros du premier conte raconté par Scheherazade, parti « s'aboucher » avec ses correspondants, et faisant une pause déjeuner au retour.

8. Les personnages des Mille et Une Nuits agissent toujours par impulsion, et doivent subir souvent dans leurs corps les effets de ces abandons; voir notre livre à paraître, Corps à coudre, LesMille et Une Nuits entre orient et occident.

\section{RÉSUMÉS}

Les effets comiques du conte merveilleux (dans l'acception large qui associe le conte de fées aux Mille et Une Nuits) affectent ce qui en détermine la logique narrative et sémiotique : la présence du surnaturel, la totale liberté à l'égard des lois de la nature et donc de la mimesis. En outre, les éléments qui servent de support à la disconvenance des logiques (réaliste/fantaisiste) ont des propriétés dont la rencontre ne produit pas seulement un effet comique, ils associent à cet effet comique un aspect bizarre, inquiétant, dérangeant, voire fantastique.

In what sense are the Arabian Nights and the classical fairy tales "comical"?

The comical effects of marvel tales (in the broad definition which associates them with the Arabian Nights) is related to the core of their narrative and semiotic logic: the presence of the supernatural, a total freedom towards the laws of nature and the rules of mimesis. The discrepancies between the various logics at work within the tales (realism/fantasy) lead to a type of encounters which not only produces comical effects, but which also associates to such comical effects eerie, unsettling, disquieting and even fantastic components.

\section{AUTEUR}

\section{JEAN-PAUL SERMAIN}

Université de Paris 3-Sorbonne nouvelle 